\title{
Analysis of Crystalline Nano Structures Embedded in Amorphous Films by Selected Area Nano Diffraction in a Cs-corrected TEM
}

\author{
* J. Yamasaki, ${ }^{*}$ S. Morishita,** N. Tanaka, ${ }^{*}$ A. Hirata, ${ }^{* * *}$ Y. Hirotsu, ${ }^{* * *}$ and T. Kato**** \\ * EcoTopia Science Institute, Nagoya University, Furo-cho, Chikusa-ku, Nagoya 464-8603, Japan \\ ** Department of Crystalline Materials Science, Nagoya University, Nagoya 464-8603, Japan \\ *** The Institute of Scientific and Industrial Research, Osaka University, Ibaraki 567-0047, Japan \\ ****Nanostructures Research Laboratory, Japan Fine Ceramics Center, Nagoya 456-8587, Japan
}

Currently, amorphous films including metallic glasses have been used as various kinds of functional materials. To understand origins of the functions and improve them, it is important that local structures in the materials are clarified at an atomic level by electron microscopy [1, 2]. In recent years, effectiveness of Cs-corrected HRTEM to the issue has been shown by observations of crystalline nano clusters embedded in an amorphous matrix of a Pd-Ni-P glass [1]. However, if size of the clusters is less than $2 \mathrm{~nm}$, it has been highly suspected that detection of their lattice fringes is hindered by amorphous parts lying above and below the clusters. In the present study, we have performed detection of crystalline nano structures embedded in a $\mathrm{Zr}_{0.6} \mathrm{Al}_{0.15} \mathrm{Ni}_{0.25}$ metallic glass by a new technique to obtain electron diffraction from a nanometer-sized area.

With respect to obtaining diffraction patterns without blurring of the spots, selected area diffraction (SAD) is more desirable than nano-probe diffraction. It is known that the drawback of SAD is areaselection-errors due to $\mathrm{Cs}$ of the objective lens, which can be reduced by Cs-correction of the imaging system. In a Cs-corrected TEM, we can obtain a diffraction pattern from a well-defined area several nanometers in diameter with parallel illumination. We refer to the technique as selected area nano diffraction (SAND), distinguishing from conventional SAD[3]. Figure 1(a) shows a hole opened in a metal thin plate by focused ion beams. The hole works as an aperture that selects a nanometer-sized area in the object plane. Figure 1(b) shows a TEM image of the hole selecting a silicon crystal with (111) lattice fringes. The corresponding diffraction pattern in Fig. 1(c) consists of sharp spots owing to parallel illumination. This feature is considered to be a great advantage for precise diffraction analyses such as electron diffractive imaging [4].

Figures 2(a)-(c) show a comparison between a traditional SAD and SAND patterns from a metallic glass of $\mathrm{Zr}_{0.6} \mathrm{Al}_{0.15} \mathrm{Ni}_{0.25}$. The diameters of the areas selected by apertures are $100 \mathrm{~nm}$ for (a) and 2.4 $\mathrm{nm}$ for (b) and (c). The halo ring in Fig. 2(a) has been replaced with many spots in Figs. 2(b) and (c). To clarify the origin of the spots, we simulated a SAND pattern from a model amorphous structure created by lattice relaxation calculations with Lennard-Jones potential. Comparing the simulated pattern (Fig.2(d)) and the experimental ones (Figs. 2(b) and (c)), it is clear that the size, the density and the distribution of the spots are reproduced well. Since the model includes only short range order (SRO) structures, it is concluded that most of the spots originate from such SRO structures. On the other hand, remarkably intense spots shown by arrows in Fig. 2(c) are suspected to come from medium range order (MRO) structures like crystalline nano clusters. Figures 2(e) and (f) show Fourier transforms of Figs. 2(b) and (c), namely two-dimensional Patterson's maps. The intense spots in Fig. 2(c) make lattice fringe-like contrast in a circled area in Fig. 2(f) as shown by arrows. The diameter of the circled area is about $3.6 \mathrm{~nm}$, which corresponds to $1.8 \mathrm{~nm}$ in real space. In Cscorrected TEM images of the region where Fig. 2(c) is obtained, lattice fringe contrast is not 
observed. It is concluded that the present method is effective for detecting crystalline nano structures less than $2 \mathrm{~nm}$ embedded in an amorphous thin film and also for measuring their sizes [5].

\section{References}

[1] Y. Hirotsu, et al., Phys. Rev. B 73 (2006) 012205.

[2] A. Hirata, et al., Mater. Trans. 48(6) (2007) 1299-1303.

[3] J. Yamasaki, et al., J. Electron Microsc. 54(2) (2005) 123-126.

[4] S. Morishita, et al., Appl. Phys. Lett. 93 (2008) 183103, and in the proceedings of this congress. [5] The authors are grateful to Prof. M. Hasegawa and Dr. H. Katoh for sample preparation. This research was partly supported by MEXT KAKENHI (18029011).
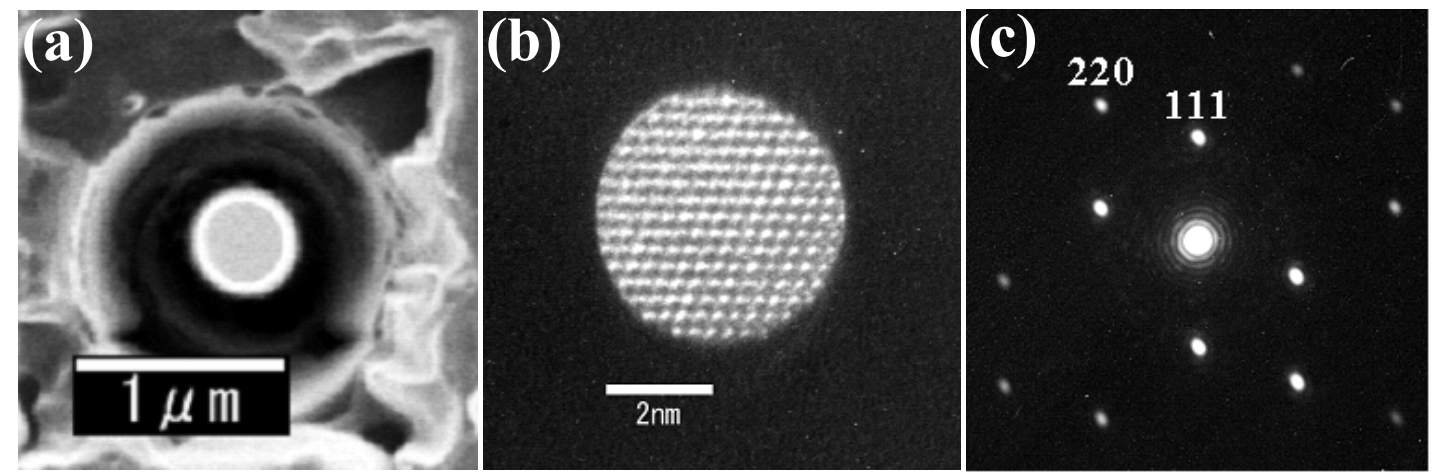

FIG. 1. (a) Ultra-fine aperture created by FIB. (b) TEM image of the aperture selecting a Si thin film. (c) Diffraction pattern corresponding to (b).
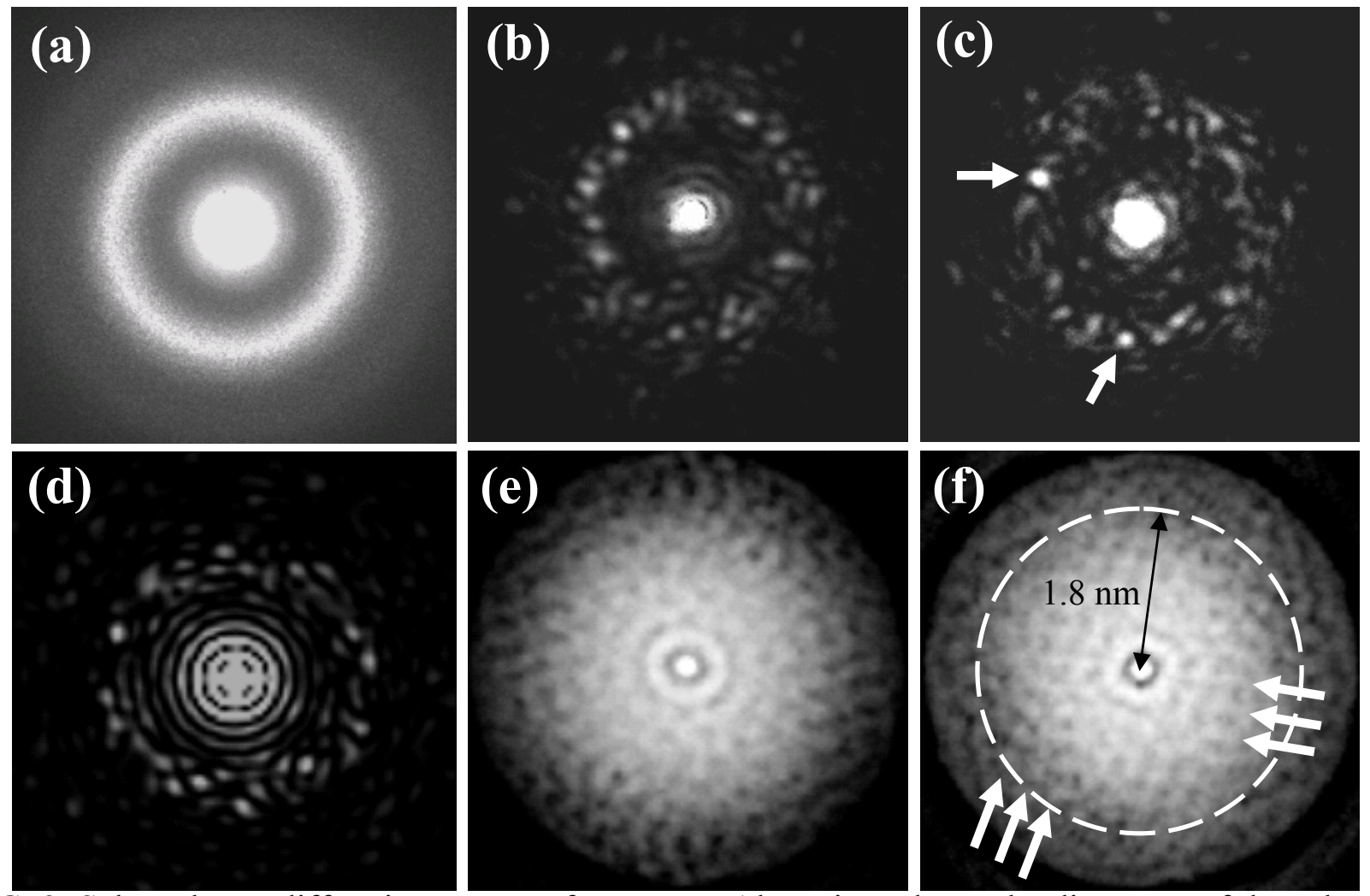

FIG. 2. Selected area diffraction patterns from a $\mathrm{Zr}_{0.6} \mathrm{Al}_{0.15} \mathrm{Ni}_{0.25}$ glass. The diameters of the selected areas are (a) $100 \mathrm{~nm}$, and (b),(c) $2.4 \mathrm{~nm}$. (d) Simulation from an amorphous structure model. (e) and (f) are Fourier transforms of (b) and (c), respectively. 